\title{
Weighted estimates for vector-valued multilinear operators with non-smooth kernels
}

\section{Zengyan $\mathrm{Si}^{*}$}

\section{"Correspondence:} zengyan@hpu.edu.cn School of Mathematics and Information Science, Henan Polytechnic University, Jiaozuo, Henan 454000, China

\begin{abstract}
Let $T$ be the multilinear Calderón-Zygmund operator with non-smooth kernel and let $T^{*}$ be its corresponding maximal operator. In this paper, vector-valued weighted norm inequalities for $T$ and $T^{*}$ are established. As applications, weighted strong type estimates for vector-valued commutators associated with $T$ and $T^{*}$ are deduced respectively.
\end{abstract}

\section{Introduction and main results}

Let $T$ be a multilinear operator initially defined on the $m$-fold product of Schwartz spaces and taking values in the space of tempered distributions,

$$
T: \mathcal{S}\left(\mathbb{R}^{n}\right) \times \cdots \times \mathcal{S}\left(\mathbb{R}^{n}\right) \rightarrow \mathcal{S}^{\prime}\left(\mathbb{R}^{n}\right)
$$

Following [1], we say that $T$ is a multilinear Calderón-Zygmund operator if, for some $1 \leq$ $q_{j}<\infty$, it extends to a bounded multilinear operator from $L^{q_{1}} \times \cdots \times L^{q_{m}}$ to $L^{q}$, where $\frac{1}{q}=\frac{1}{q_{1}}+\cdots+\frac{1}{q_{m}}$, and if there exists a function $K$, defined off the diagonal $x=y_{1}=\cdots=y_{m}$ in $\left(\mathbb{R}^{n}\right)^{m+1}$, satisfying

$$
T(\vec{f})(x)=T\left(f_{1}, \ldots, f_{m}\right)(x)=\int_{\left(\mathbb{R}^{n}\right)^{m}} K\left(x, y_{1}, \ldots, y_{m}\right) f_{1}\left(y_{1}\right) \cdots f_{m}\left(y_{m}\right) d y_{1} \cdots d y_{m}
$$

for all $x \notin \bigcap_{j=1}^{m} \operatorname{supp} f_{j}$;

$$
\left|K\left(y_{0}, y_{1}, \ldots, y_{m}\right)\right| \leq \frac{A}{\left(\sum_{k, l=0}^{m}\left|y_{k}-y_{l}\right|\right)^{m n}}
$$

and

$$
\left|K\left(y_{0}, y_{1}, \ldots, y_{j}, \ldots, y_{m}\right)-K\left(y_{0}, y_{1}, \ldots, y_{j}^{\prime}, \ldots, y_{m}\right)\right| \leq \frac{A\left|y_{j}-y_{j}^{\prime}\right|^{\epsilon}}{\left(\sum_{k, l=0}^{m}\left|y_{k}-y_{l}\right|\right)^{m n+\epsilon}}
$$

for some $\epsilon>0$ and all $0 \leq j \leq m$, whenever $2\left|y_{j}-y_{j}^{\prime}\right| \leq \max _{0 \leq k \leq m}\left|y_{j}-y_{k}\right|$. Such kernels are called $m$-linear Calderón-Zygmund kernels, and the collection of such functions is

(c) 2013 Si; licensee Springer. This is an Open Access article distributed under the terms of the Creative Commons Attribution License (http://creativecommons.org/licenses/by/2.0), which permits unrestricted use, distribution, and reproduction in any medium, provided the original work is properly cited. 
denoted by $m-C Z K(A, \epsilon)$ in [1]. As in [2], we define the maximal multilinear operator by

$$
T^{*}(\vec{f})(x)=\sup _{\delta>0}\left|T_{\delta}\left(f_{1}, \ldots, f_{m}\right)(x)\right|
$$

where $T_{\delta}$ is the smooth truncation of $T$ given by

$$
T_{\delta}(\vec{f})(x)=\int_{\left|x-y_{1}\right|^{2}+\cdots+\left|x-y_{m}\right|^{2}>\delta^{2}} K\left(x, y_{1}, \ldots, y_{m}\right) f_{1}\left(y_{1}\right) \cdots f_{m}\left(y_{m}\right) d y_{1} \cdots d y_{m}
$$

The vector-valued multilinear Calderón-Zygmund operator $T_{q}$ and vector-valued maximal multilinear operator $T_{q}^{*}$ associated with $T$ are defined and studied in $[3,4]$.

$$
\begin{aligned}
T_{q}(\vec{f})(x) & =\left|T\left(f_{1}, \ldots, f_{m}\right)(x)\right|_{q}=\left\|T\left(f_{1}, \ldots, f_{m} .\right)(x)\right\|_{l q} \\
& =\left(\sum_{k=1}^{\infty}\left|T\left(f_{1 k}, \ldots, f_{m k}\right)(x)\right|^{q}\right)^{1 / q}, \\
T_{q}^{*}(\vec{f})(x) & =\left|T^{*}\left(f_{1}, \ldots, f_{m}\right)(x)\right|_{q}=\left\|T^{*}\left(f_{1}, \ldots, f_{m} .\right)(x)\right\|_{l^{q}} \\
& =\left(\sum_{k=1}^{\infty}\left|T^{*}\left(f_{1 k}, \ldots, f_{m k}\right)(x)\right|^{q}\right)^{1 / q},
\end{aligned}
$$

where $f_{i}=\left\{f_{i k}\right\}_{k=1}^{\infty}$ for $i=1, \ldots, m$.

Theorem A [3] Assume that $T$ is a multilinear Calderón-Zygmund operator. Let $1<$ $p_{1}, \ldots, p_{m}<\infty, 1<q_{1}, \ldots, q_{m}<\infty$ and $1 / m<p, q<\infty$ such that $\frac{1}{p}=\frac{1}{p_{1}}+\cdots+\frac{1}{p_{m}}, \frac{1}{q}=$ $\frac{1}{q_{1}}+\cdots+\frac{1}{q_{m}}$.If $\left(\omega_{1}^{p_{1}}, \ldots, \omega_{m}^{p_{m}}\right) \in\left(A_{p_{1}}, \ldots, A_{p_{m}}\right)$, then there exists a constant $C>0$ such that

$$
\left\|T_{q}(\vec{f})\right\|_{L^{p}\left(\omega_{1}^{p} \ldots \omega_{m}^{p}\right)} \leq C \prod_{j=1}^{m}\left\|\left|f_{j}\right|_{q_{j}}\right\|_{L^{p_{j}}\left(\omega_{j}^{p_{j}}\right)} .
$$

Theorem B [4] Assume that $T$ is a multilinear Calderón-Zygmund operator. Let $1 \leq$ $p_{1}, \ldots, p_{m}<\infty, 1<q_{1}, \ldots, q_{m}<\infty$ and $0<p, q<\infty$ such that $\frac{1}{p}=\frac{1}{p_{1}}+\cdots+\frac{1}{p_{m}}, \frac{1}{q}=$ $\frac{1}{q_{1}}+\cdots+\frac{1}{q_{m}}$.

(i) If $1<p_{1}, \ldots, p_{m}<\infty$ and $\omega \in A_{p_{1}} \cap \cdots \cap A_{p_{m}}$, then there exists a constant $C>0$ such that

$$
\left\|T_{q}(\vec{f})\right\|_{L^{p}(\omega)} \leq C \prod_{j=1}^{m}\left\|\left|f_{j}\right|_{q_{j}}\right\|_{L^{p_{j}(\omega)}} .
$$

(ii) If at least one $p_{j}=1$ and $\omega \in A_{1}$, then there exists a constant $C>0$ such that

$$
\left\|T_{q}(\vec{f})\right\|_{L^{p, \infty}(\omega)} \leq C \prod_{j=1}^{m}\left\|\left|f_{j}\right|_{q_{j}}\right\|_{L^{p_{j}(\omega)}} .
$$

It is worth noting that similar results hold for $T^{*}$ in Theorems A and B. 
We will replace (1.3) by a weaker regularity condition on the kernel $K$. Assume that operators $A_{t}$ are associated with kernels $a_{t}(x, y)$ in the sense that

$$
A_{t} f(x)=\int_{\mathbb{R}^{n}} a_{t}(x, y) f(y) d y
$$

for every function $f \in L^{p}\left(\mathbb{R}^{n}\right), 1 \leq p \leq \infty$, and $a_{t}(x, y)$ satisfy the following size condition:

$$
\left|a_{t}(x, y)\right| \leq h_{t}(x, y)=t^{-n / s} h\left(\frac{|x-y|^{s}}{t}\right),
$$

where $s$ is a positive fixed constant and $h$ is a positive, bounded, decreasing function satisfying that for some $\eta>0$,

$$
\lim _{r \rightarrow \infty} r^{n+\eta} h\left(r^{s}\right)=0 .
$$

The $j$ th transpose $T^{*, j}$ of $T$ is defined via

$$
\left\langle T^{*, j}\left(f_{1}, \ldots, f_{m}\right), g\right\rangle=\left\langle T\left(f_{1}, \ldots, f_{j-1}, g, f_{j+1}, \ldots, f_{m}\right), f_{j}\right\rangle
$$

for all $f_{1}, \ldots, f_{m}, g$ in $\mathcal{S}\left(\mathbb{R}^{n}\right)$. It is easy to check that the kernel $K^{*, j}$ of $T^{*, j}$ is related to the kernel $K$ of $T$ via the identity

$$
K^{*, j}\left(x, y_{1}, \ldots, y_{j-1}, y_{j}, y_{j+1}, \ldots, y_{m}\right)=K\left(y_{j}, y_{1}, \ldots, y_{j-1}, x, y_{j+1}, \ldots, y_{m}\right) .
$$

To maintain uniform notation, we may occasionally denote $T=T^{*, 0}$ and $K=K^{*, 0}$.

Assumption (H0) We always assume that there exist some $1 \leq q_{1}, \ldots, q_{m}<\infty$ and some $0<q<\infty$ with $\frac{1}{q}=\frac{1}{q_{1}}+\cdots+\frac{1}{q_{m}}$ such that both $T^{*}$ and $T$ map $L^{q_{1}} \times \cdots \times L^{q_{m}}$ to $L^{q, \infty}$.

Assumption (H1) Assume that there exist operators $\left\{A_{t}^{(i)}\right\}_{t>0}$ with kernels $a_{t}^{(i)}(x, y)$ that satisfy the conditions (1.4) and (1.5) with constants $s$ and $\eta$ for each $i=1, \ldots, m$ and that for every $j=0,1,2, \ldots, m$, there exists kernel $K_{t}^{*, j, i}\left(x, y_{1}, \ldots, y_{m}\right)$ such that

$$
\begin{aligned}
& \left\langle T^{*, j}\left(f_{1}, \ldots, A_{t}^{(i)} f_{i}, \ldots, f_{m}\right), g\right\rangle \\
& \quad=\int_{\mathbb{R}^{n}} \int_{\left(\mathbb{R}^{n}\right)^{m}} K_{t}^{*, j,(i)}\left(x, y_{1}, \ldots, y_{m}\right) f_{1}\left(y_{1}\right) \cdots f_{m}\left(y_{m}\right) g(x) d y_{1} \cdots d y_{m} d x,
\end{aligned}
$$

for all $f_{1}, \ldots, f_{m}$ in $\mathcal{S}\left(\mathbb{R}^{n}\right)$ with $\bigcap_{k=1}^{m} \operatorname{supp} f_{k} \cap \operatorname{supp} g=\phi$. Also assume that there exist a nonnegative function $\phi \in C(\mathbb{R})$ with $\operatorname{supp} \phi \in[-1,1]$ and a constant $\epsilon>0$ so that for every $j \in\{0,1, \ldots, m\}$ and every $i \in\{1,2, \ldots, m\}$, all $t>0$ and all $x, y_{1}, \ldots, y_{n} \in \mathbb{R}^{n}$, we have

$$
\begin{aligned}
& \left|K^{*, j}\left(x, y_{1}, \ldots, y_{m}\right)-K_{t}^{*, j,(i)}\left(x, y_{1}, \ldots, y_{m}\right)\right| \\
& \leq \frac{A}{\left(\left|x-y_{1}\right|+\cdots+\left|x-y_{m}\right|\right)^{m n}} \sum_{k=1, k \neq i}^{m} \phi\left(\frac{\left|y_{i}-y_{k}\right|}{t^{1 / s}}\right) \\
& \quad+\frac{A t^{\epsilon / s}}{\left(\left|x-y_{1}\right|+\cdots+\left|x-y_{m}\right|\right)^{m n+\epsilon}},
\end{aligned}
$$

whenever $2 t^{1 / s} \leq\left|x-y_{i}\right|$. 
Kernels $K$ that satisfy the size estimate (1.2) and Assumption (H1) with parameters $m$, $A, s, \eta, \varepsilon$ are called generalized Calderón-Zygmund kernels, and their collection is denoted by $m-\operatorname{GCZK}(A, s, \eta, \varepsilon)$. We say that $T$ is of class $m-\operatorname{GCZO}(A, s, \eta, \varepsilon)$ if $T$ has an associated kernel $K$ in $m-G C Z K(A, s, \eta, \varepsilon)$.

Assumption (H2) Assume that there exist operators $\left\{A_{t}\right\}_{t>0}$ with kernels $a_{t}(x, y)$ that satisfy conditions (1.4) and (1.5) with constants $s$ and $\eta$, and there exist kernels $K_{t}^{(0)}\left(x, y_{1}\right.$, $\left.\ldots, y_{m}\right)$ such that the representation is valid

$$
K_{t}^{(0)}\left(x, y_{1}, \ldots, y_{m}\right)=\int_{\mathbb{R}^{n}} K\left(z, y_{1}, \ldots, y_{m}\right) a_{t}(x, z) d z
$$

and that there exist a non-negative function $\phi \in \mathcal{C}(\mathbb{R})$ and $\operatorname{supp} \phi \subset[-1,1]$ and a positive constant $\varepsilon$ such that

$$
\begin{aligned}
& \left|K\left(x, y_{1}, \ldots, y_{m}\right)-K_{t}^{(0)}\left(x, y_{1}, \ldots, y_{m}\right)\right| \\
& \quad \leq \frac{A}{\left(\sum_{k=1}^{m}\left|x-y_{k}\right|\right)^{m n}} \sum_{\substack{k=1 \\
k \neq j}}^{m} \phi\left(\frac{\left|x-y_{k}\right|}{t^{1 / s}}\right)+\frac{A t^{\varepsilon / s}}{\left(\sum_{k=1}^{m}\left|x-y_{k}\right|\right)^{m n+\varepsilon}}
\end{aligned}
$$

for some $A>0$, whenever $2 t^{1 / s} \leq \max _{1 \leq j \leq m}\left|x-y_{j}\right|$. Moreover, assume that for all $x, y_{1}, \ldots, y_{m} \in \mathbb{R}^{n}$,

$$
\left|K_{t}^{(0)}\left(x, y_{1}, \ldots, y_{m}\right)\right| \leq \frac{A}{\left(\sum_{k=1}^{m}\left|x-y_{k}\right|\right)^{m n}}
$$

whenever $2 t^{1 / s} \leq \min _{1 \leq j \leq m}\left|x-y_{j}\right|$, and for all $x, x^{\prime}, y_{1}, \ldots, y_{m} \in \mathbb{R}^{n}$,

$$
\left|K\left(x, y_{1}, \ldots, y_{m}\right)-K_{t}^{(0)}\left(x^{\prime}, y_{1}, \ldots, y_{m}\right)\right| \leq \frac{A t^{\varepsilon / s}}{\left(\sum_{k=1}^{m}\left|x-y_{k}\right|\right)^{m n+\varepsilon}},
$$

whenever $2 t^{1 / s} \leq \min _{1 \leq j \leq m}\left|x-y_{j}\right|$ and $2\left|x-x^{\prime}\right| \leq t^{1 / s}$.

The commutators associated with $T$ and $T^{*}$ are defined respectively by

$$
\begin{aligned}
T_{\Pi \vec{b}}(\vec{f})(x) & =\left[b_{1},\left[b_{2}, \ldots\left[b_{l-1},\left[b_{l}, T\right]_{l}\right]_{l-1} \ldots\right]_{2}\right]_{1}(\vec{f})(x) \\
& =\int_{\left(\mathbb{R}^{n}\right)^{m}} \prod_{j=1}^{l}\left(b_{j}(x)-b_{j}\left(y_{j}\right)\right) K\left(x, y_{1}, \ldots, y_{m}\right) \prod_{i=1}^{m} f_{i}\left(y_{i}\right) d \vec{y},
\end{aligned}
$$

and

$$
\begin{aligned}
T_{\Pi \vec{b}}^{*}(\vec{f})(x) & =\sup _{\delta>0}\left|\left[b_{1},\left[b_{2}, \ldots\left[b_{l-1},\left[b_{l}, T_{\delta}\right]_{l}\right]_{l-1} \ldots\right]_{2}\right]_{1}(\vec{f})(x)\right| \\
& =\sup _{\delta>0}\left|\int_{\left|x-y_{1}\right|^{2}+\cdots+\left|x-y_{m}\right|^{2}>\delta^{2}} \prod_{j=1}^{l}\left(b_{j}(x)-b_{j}\left(y_{j}\right)\right) K\left(x, y_{1}, \ldots, y_{m}\right) \prod_{i=1}^{m} f_{i}\left(y_{i}\right) d \vec{y}\right| .
\end{aligned}
$$

Here and subsequently, we often write $\vec{y}=\left(y_{1}, \ldots, y_{m}\right)$ and $d \vec{y}=d y_{1} \cdots d y_{m}$.

For simplicity of notation, we often write $\vec{f}=\left(f_{1}, \ldots, f_{m}\right)$ with $f_{j}=\left\{f_{j k}\right\}_{k=1}^{\infty}$. For the sequence $\left\{\vec{f}_{k}\right\}_{k=1}^{\infty}=\left\{f_{1 k}, \ldots, f_{m k}\right\}_{k=1}^{\infty}$ of vector functions, the commutators associated with 
vector-valued $T_{q}$ and $T_{q}^{*}$ can be defined by

$$
\begin{aligned}
& T_{\Pi \vec{b}, q}(\vec{f})(x)=\left|T_{\Pi \vec{b}}(\vec{f})(x)\right|_{q}=\left\|T_{\Pi \vec{b}}\left(f_{1}, \ldots, f_{m} .\right)(x)\right\|_{l q}=\left(\sum_{k=1}^{\infty}\left|T_{\Pi \vec{b}}\left(\vec{f}_{k}\right)(x)\right|^{q}\right)^{1 / q}, \\
& T_{\Pi \vec{b}, q}^{*}(\vec{f})(x)=\left|T_{\Pi \vec{b}}^{*}(\vec{f})(x)\right|_{q}=\left\|T_{\Pi \vec{b}}^{*}\left(f_{1}, \ldots, f_{m} .\right)(x)\right\|_{l q}=\left(\sum_{k=1}^{\infty}\left|T_{\Pi \vec{b}}^{*}\left(\vec{f}_{k}\right)(x)\right|^{q}\right)^{1 / q} .
\end{aligned}
$$

From now on, we always assume that $T$ is a multilinear operator in $m-G C Z O(A, s, \eta, \varepsilon)$ and its kernel satisfies Assumption (H2). Recently, if $l=m$, Peng et al. [5] obtained the following weighted strong type estimates for $T_{\Pi \vec{b}}$ and $T_{\Pi \vec{b}}^{*}$ with multiple weights (see Definition 2.1).

Theorem C [5] Let $\vec{b} \in B M O^{m}, \frac{1}{p}=\frac{1}{p_{1}}+\cdots+\frac{1}{p_{m}}$ with $1<p_{j}<\infty, j=1, \ldots, m$. Then we have

(i) There exists a constant $C$ such that

$$
\left\|T_{\Pi \vec{b}}^{*}(\vec{f})\right\|_{L^{p}\left(\nu_{\vec{\omega}}\right)} \leq C \prod_{i=1}^{m}\left\|b_{i}\right\|_{B M O} \prod_{i=1}^{m}\left\|f_{i}\right\|_{L^{p_{i}\left(M \omega_{i}\right)}} .
$$

(ii) If each $\omega_{i} \in A_{p_{j}}$, then there exists a constant $C$ such that

$$
\left\|T_{\Pi \vec{b}}^{*}(\vec{f})\right\|_{L^{p}\left(\nu_{\vec{\omega}}\right)} \leq C \prod_{i=1}^{m}\left\|b_{i}\right\|_{B M O} \prod_{i=1}^{m}\left\|f_{i}\right\|_{L^{p_{i}\left(\omega_{i}\right)}}
$$

where $v_{\vec{\omega}}=\prod_{i=1}^{m} \omega_{i}^{p / p_{j}}$. Similar results still hold for $T_{\Pi \vec{b}}$, which extend the results in [6] significantly.

In this work, we first pursue results parallel to Theorems A and B, then extend Theorem $C$ to a vector-valued version. The main results can be stated as follows.

Theorem 1.1 Let $1<p_{1}, \ldots, p_{m}<\infty, 1<q_{1}, \ldots, q_{m}<\infty$ and $1 / m<p, q<\infty$ such that $\frac{1}{p}=$ $\frac{1}{p_{1}}+\cdots+\frac{1}{p_{m}}, \frac{1}{q}=\frac{1}{q_{1}}+\cdots+\frac{1}{q_{m}}$. If $\left(\omega_{1}^{p_{1}}, \ldots, \omega_{m}^{p_{m}}\right) \in\left(A_{p_{1}}, \ldots, A_{p_{m}}\right)$, then there exists a constant $C>0$ such that

$$
\left\|T_{q}(\vec{f})\right\|_{L^{p}\left(\omega_{1}^{p} \cdots \omega_{m}^{p}\right)} \leq C \prod_{j=1}^{m}\left\|\left|f_{j}\right|_{q_{j}}\right\|_{L^{p_{j}}\left(\omega_{j}^{p_{j}}\right)} .
$$

Moreover, similar estimates hold for $T^{*}$.

Theorem 1.2 Let $1 \leq p_{1}, \ldots, p_{m}<\infty, 1<q_{1}, \ldots, q_{m}<\infty$ and $0<p, q<\infty$ such that $\frac{1}{p}=$ $\frac{1}{p_{1}}+\cdots+\frac{1}{p_{m}}, \frac{1}{q}=\frac{1}{q_{1}}+\cdots+\frac{1}{q_{m}}$.

(i) If $1<p_{1}, \ldots, p_{m}<\infty$ and $\omega \in A_{p_{1}} \cap \cdots \cap A_{p_{m}}$, then there exists a constant $C>0$ such that

$$
\left\|T_{q}(\vec{f})\right\|_{L^{p}(\omega)} \leq C \prod_{j=1}^{m}\left\|\left|f_{j}\right|_{q_{j}}\right\|_{L^{p_{j}(\omega)}}
$$


(ii) If at least one $p_{j}=1$ and $\omega \in A_{1}$, then there exists a constant $C>0$ such that

$$
\left\|T_{q}(\vec{f})\right\|_{L^{p, \infty}(\omega)} \leq C \prod_{j=1}^{m}\left\|\left|f_{j}\right|_{q_{j}}\right\|_{L^{p_{j}(\omega)}} .
$$

Moreover, similar estimates hold for $T^{*}$.

Theorem 1.3 Let $1 / m<p<\infty, \frac{1}{p}=\frac{1}{p_{1}}+\cdots+\frac{1}{p_{m}}$ with $1<p_{1}, \ldots, p_{m}<\infty, 1 / m<q<\infty$, and $\frac{1}{q_{1}}+\cdots+\frac{1}{q_{m}}=\frac{1}{q}$ with $1<q_{1}, \ldots, q_{m}<\infty$. Suppose that $\vec{\omega} \in A_{\vec{p}}, v_{\vec{\omega}}=\prod_{i=1}^{m} \omega_{i}^{\frac{p}{p_{i}}}$ and $\vec{b} \in(B M O)^{l}$. Then we have

(i) There then exists a constant $C>0$ such that

$$
\left\|T_{\Pi \vec{b}, q}(\vec{f})\right\|_{L^{p}\left(v_{\overparen{\omega})}\right)} \leq \prod_{j=1}^{l}\left\|b_{j}\right\|_{B M O} \prod_{j=1}^{m}\left\|\left|f_{j}\right|_{q_{j}}\right\|_{L^{p_{j}\left(M w_{j}\right)}} .
$$

(ii) If $\omega_{j} \in A_{p_{j}}$, then there exists a constant $C>0$ such that

$$
\left\|T_{\Pi \vec{b}, q}(\vec{f})\right\|_{L^{p}\left(v_{\vec{\omega}}\right)} \leq \prod_{j=1}^{l}\left\|b_{j}\right\|_{B M O} \prod_{j=1}^{m}\left\|\left|f_{j}\right|_{q_{j}}\right\|_{L^{p_{j}}\left(w_{j}\right)} .
$$

Moreover, similar estimates hold for $T^{*}$.

Remark 1.4 If $l=1$ and $l=m$, Theorem 1.3 can be seen as the vector-valued extension of Theorem 4.5 in [6] and Theorem C, respectively.

\section{Proofs of Theorem 1.1 and Theorem 1.2}

Let us begin with the definition of Hardy-Littlewood maximal operator, that is

$$
M f(x)=\sup _{Q \ni x} \frac{1}{|Q|} \int_{Q}|f(y)| d y .
$$

The sharp maximal function is defined by

$$
M^{\sharp} f(x)=\operatorname{supinf}_{Q \ni x} \frac{1}{|Q|} \int_{Q}|f(y)-c| d y \approx \sup _{Q \ni x} \frac{1}{|Q|} \int_{Q}\left|f(y)-f_{Q}\right| d y .
$$

For $\delta>0$, we also need the maximal function $M_{\delta} f=M\left(|f|^{\delta}\right)^{\frac{1}{\delta}}$ and $M_{\delta}^{\sharp} f=M^{\sharp}\left(|f|^{\delta}\right)^{\frac{1}{\delta}}$.

The new maximal function $\mathcal{M}$ can be defined by

$$
\mathcal{M}(\vec{f})(x)=\sup _{Q \ni x} \prod_{j=1}^{m} \frac{1}{|Q|} \int_{Q}\left|f_{j}\left(y_{j}\right)\right| d y_{j}
$$

For $1 \leq l \leq m$, as in [7], a modified maximal function $\mathcal{M}_{l}$ is given by

$$
\mathcal{M}_{l}(\vec{f})(x)=\sup _{Q \ni x} \sum_{k=0}^{\infty} 2^{-k n l}\left(\prod_{j=1}^{l} \frac{1}{|Q|} \int_{Q}\left|f_{j}\left(y_{j}\right)\right| d y_{j}\right)\left(\prod_{j=l+1}^{m} \frac{1}{\left|2^{k} Q\right|} \int_{2^{k} Q}\left|f_{j}\left(y_{j}\right)\right| d y_{j}\right) .
$$


For exponents $p_{1}, \ldots, p_{m}$, we will often write $p$ for the number given by $1 / p=1 / p_{1}+\cdots+$ $1 / p_{m}$, and $\vec{p}$ for the vector $\vec{p}=\left(p_{1}, \ldots, p_{m}\right)$. Let us recall the definition of $A_{\vec{p}}$ weights.

Definition 2.1 Let $1 \leq p_{1}, \ldots, p_{m}<\infty$. Given $\vec{\omega}=\left(\omega_{1}, \ldots, \omega_{m}\right)$, set $v_{\vec{\omega}}=\prod_{i=1}^{m} \omega_{i}^{p / p_{i}}$. We say that $\vec{\omega}$ satisfies the $A_{\vec{p}}$ condition if

$$
\sup _{Q}\left(\frac{1}{|Q|} \int_{Q} \prod_{i=1}^{m} \omega_{i}^{\frac{p}{p_{i}}}\right)^{\frac{1}{p}} \prod_{i=1}^{m}\left(\frac{1}{|Q|} \int_{Q} \omega_{i}^{1-p_{i}^{\prime}}\right)^{\frac{1}{p_{i}^{\prime}}}<\infty
$$

when $p_{i}=1,\left(\frac{1}{|Q|} \int_{Q} \omega_{i}^{1-p_{i}^{\prime}}\right)^{\frac{1}{p_{i}^{\prime}}}$ is understood as $\left(\inf _{Q} \omega_{i}\right)^{-1}$.

We will use the following lemmas in the proof of Theorem 1.1 and Theorem 1.2.

Lemma 2.2 [3] Let $\mathcal{T}$ be an m-linear operator, and let $1<q_{1}, \ldots, q_{m}<\infty$ and $\frac{1}{m}<q<\infty$ be fixed indices such that $\frac{1}{q}=\frac{1}{q_{1}}+\cdots+\frac{1}{q_{m}}$. For $\left(\omega_{1}^{q_{1}}, \ldots, \omega_{m}^{q_{m}}\right) \in\left(A_{q_{1}}, \ldots, A_{q_{m}}\right)$, the following estimate holds:

$$
\|\mathcal{T}(\vec{f})\|_{L^{q}\left(\omega_{1}^{q} \cdots \omega_{m}^{q}\right)} \leq C \prod_{j=1}^{m}\left\|f_{j}\right\|_{L^{q_{j}}\left(\omega_{j}^{q_{j}}\right)}
$$

Then, for all indices, $1<p_{1}, \ldots, p_{m}<\infty$ and $\frac{1}{m}<p<\infty$ satisfy $\frac{1}{p}=\frac{1}{p_{1}}+\cdots+\frac{1}{p_{m}}$, $1<s_{1}, \ldots, s_{m}<\infty$ and $\frac{1}{m}<s<\infty$ such that $\frac{1}{s}=\frac{1}{s_{1}}+\cdots+\frac{1}{s_{m}}$, and all $\left(\omega_{1}^{p_{1}}, \ldots, \omega_{m}^{p_{m}}\right) \in$ $\left(A_{p_{1}}, \ldots, A_{p_{m}}\right)$. Then the following inequality holds:

$$
\left\|\left(\sum_{k}\left|\mathcal{T}\left(f_{1 k}, \ldots, f_{m k}\right)\right|^{s}\right)^{\frac{1}{s}}\right\|_{L^{p}\left(\omega_{1}^{p} \ldots \omega_{m}^{p}\right)} \leq C \prod_{j=1}^{m}\left\|\left(\sum_{k}\left|f_{j k}\right|^{s_{j}}\right)^{\frac{1}{s_{j}}}\right\|_{L^{p_{j}\left(\omega_{j}^{p_{j}}\right)}} .
$$

Lemma 2.3 [7] Suppose that for some $1 \leq q_{1}, q_{2}, \ldots, q_{m-1} \leq \infty, q_{m} \in(1, \infty)$ and $q \in(0, \infty)$ satisfying $\frac{1}{q}=\frac{1}{q_{1}}+\cdots+\frac{1}{q_{m}}$, T maps $L^{q_{1}} \times \cdots \times L^{q_{m}}$ to $L^{q}$. Let $1 \leq p_{1}, \ldots, p_{m}<\infty, \frac{1}{p}=$ $\frac{1}{p_{1}}+\cdots+\frac{1}{p_{m}}, \vec{\omega}=\left(\omega_{1}, \ldots, \omega_{m}\right) \in A_{\vec{p}}$ and $\vec{p}=\left(p_{1}, \ldots, p_{m}\right)$. Then

(i) $T^{*}$ can be extended to a bounded operator from $L^{p_{1}}\left(\omega_{1}\right) \times \cdots \times L^{p_{m}}\left(\omega_{m}\right)$ to $L^{p}\left(v_{\vec{\omega}}\right)$ if all the exponents $p_{j}$ are strictly greater than 1.

(ii) $T^{*}$ can be extended to a bounded operator from $L^{p_{1}}\left(\omega_{1}\right) \times \cdots \times L^{p_{m}}\left(\omega_{m}\right)$ to $L^{p, \infty}\left(v_{\vec{\omega}}\right)$ if some exponents $p_{j}$ equal 1.

Similar results hold for $T$.

Note that if each $\omega_{j} \in A_{p_{j}}$, then $\prod_{j=1}^{m} A_{p_{j}} \subset A_{\vec{p}}$ and this inclusion is strict (see [8] for details). This fact together with Lemma 2.3 yields the following weighted estimates.

Lemma 2.4 Consider an m-tuple $\left(\omega_{1}^{p_{1}}, \ldots, \omega_{m}^{p_{m}}\right) \in\left(A_{p_{1}}, \ldots, A_{p_{m}}\right)$, where $1<p_{1}, \ldots, p_{m}<\infty$ and $\frac{1}{m}<p<\infty$ satisfy $\frac{1}{p}=\frac{1}{p_{1}}+\cdots+\frac{1}{p_{m}}$. Then there exists a constant $C$ such that

$$
\|T \overrightarrow{(f)}\|_{L^{p}\left(\omega_{1}^{p} \ldots \omega_{m}^{p}\right)} \leq C \prod_{j=1}^{m}\left\|f_{j}\right\|_{L^{p_{j}}\left(\omega_{j}^{p_{j}}\right)}
$$


and

$$
\left\|T^{*}(\vec{f})\right\|_{L^{p}\left(\omega_{1}^{p} \cdots \omega_{m}^{p}\right)} \leq C \prod_{j=1}^{m}\left\|f_{j}\right\|_{L^{p_{j}}\left(\omega_{j}^{p_{j}}\right)}
$$

Proof of Theorem 1.1 and Theorem 1.2 As a consequence of Lemma 2.2 and Lemma 2.4, we obtain Theorem 1.1 (see the proof of Corollary 3 in [3]). From [7] we know that for all $1 \leq l \leq m, \vec{f}=\left(f_{1}, \ldots, f_{m}\right)$ and $x \in \mathbb{R}^{n}$, the following two inequalities hold:

$$
\begin{aligned}
& \mathcal{M}(\vec{f})(x) \leq \mathcal{M}_{l}(\vec{f})(x) \leq 2 \prod_{j=1}^{m} M\left(f_{j}\right)(x), \\
& \|T \vec{f}\|_{L^{p}\left(v_{\vec{\omega}}\right)} \leq C\left\|\sum_{l=1}^{m} \mathcal{M}_{l}(\vec{f})\right\|_{L^{p}\left(v_{\vec{\omega}}\right)} .
\end{aligned}
$$

So, we get $\|T \vec{f}\|_{L^{p}\left(v_{\vec{\omega}}\right)} \leq C\left\|\prod_{j=1}^{m} M\left(f_{j}\right)\right\|_{L^{p}\left(v_{\vec{\omega}}\right)}$. A similar inequality still holds for $T^{*}$. Theorem 1.2 follows by repeating the same steps as in Corollary 3.3 in [4]. In fact, we apply Theorem 2.1 in [4] to the families

$$
\mathcal{F}\left(T\left(f_{1}, \ldots, f_{m}\right), \prod_{j=1}^{m} M f_{j}\right), \quad \mathcal{F}\left(T^{*}\left(f_{1}, \ldots, f_{m}\right), \prod_{j=1}^{m} M f_{j}\right) .
$$

Hölder's inequality and the normal inequalities for the maximal operator yield the desired results.

\section{Proof of Theorem 1.3}

We begin with some lemmas which will be used in the proof of Theorem 1.3.

Lemma 3.1 [9] Let $0<p, \delta<\infty$ and let $\omega$ be a weight in $A_{\infty}$. Then there exists $C>0$ (depending upon the $A_{\infty}$ condition of $\omega$ ) such that

$$
\int_{\mathbb{R}^{n}}\left(M_{\delta} f(x)\right)^{p} \omega(x) d x \leq C \int_{\mathbb{R}^{n}}\left(M_{\delta}^{\sharp} f(x)\right)^{p} \omega(x) d x
$$

for every function such that the left-hand side is finite.

Lemma 3.2 Let $0<\delta<1 / m, 1 / m<q<\infty$ and $1 / q=1 / q_{1}+\cdots+1 / q_{m}$ with $1<q_{1}, \ldots, q_{m}<\infty$. Then there exists a constant $C>0$ such that

$$
M_{\delta}^{\sharp}\left(T_{q}(\vec{f})\right)(x) \leq C \prod_{j=1}^{m} M\left(\left|f_{j}\right|_{q_{j}}\right)(x)
$$

for any smooth vector function $\left\{\vec{f}_{k}\right\}_{k=1}^{\infty}$ for any $x \in \mathbb{R}^{n}$.

Proof Fix a point $x \in \mathbb{R}^{n}$ and a cube $Q$ centered at $x$. Set $\vec{f}_{j}=\vec{f}_{j}^{0}+\vec{f}_{j}^{\infty}$, where $\vec{f}_{j}^{0}=\vec{f}_{j} \chi_{Q^{*}}$. Let $\vec{f}^{\alpha}=f_{1}^{\alpha_{1}} \cdots f_{m}^{\alpha_{m}}$ and $Q^{*}=(8 \sqrt{n}+4) Q$. It is easy to see

$$
\left|T_{q}(\vec{f})(z)-\mathcal{C}\right| \leq\left|T_{q}\left(\vec{f}^{0}\right)(z)\right|+\sum_{\alpha_{1}, \ldots, \alpha_{m}}\left|T\left(\vec{f}^{\alpha}\right)(z)-T\left(\vec{f}^{\alpha}\right)(x)\right|_{q^{\prime}}
$$


where $\mathcal{C}=\sum_{\alpha_{1}, \ldots, \alpha_{m}}\left|T\left(f_{1}^{\alpha_{1}} \cdots f_{m}^{\alpha_{m}}\right)(x)\right|_{q}$ and in the last sum each $\alpha_{j}=0$ or $\infty$ and in each term there is at least one $\alpha_{j}=\infty$. Since $0<\delta<1 / m<1$, it follows that

$$
\begin{aligned}
& \left(\left.\frac{1}{|Q|} \int_{Q}|| T_{q}(\vec{f})(z)\right|^{\delta}-|\mathcal{C}|^{\delta} \mid d z\right)^{1 / \delta} \\
& \quad \leq C\left(\frac{1}{|Q|} \int_{Q}|T(\vec{f})(z)-c|_{q}^{\delta} d z\right)^{1 / \delta} \\
& \quad \leq C\left(\frac{1}{|Q|} \int_{Q}\left|T\left(\vec{f}^{0}\right)(z)\right|_{q}^{\delta} d z\right)^{1 / \delta}+C \sum_{\alpha_{1}, \ldots, \alpha_{m}}\left(\frac{1}{|Q|} \int_{Q}\left|T\left(\vec{f}^{\alpha}\right)(z)-c\right|_{q}^{\delta} d z\right)^{1 / \delta} \\
& \quad \triangleq P_{1}+P_{2},
\end{aligned}
$$

where $\mathcal{C}=|c|_{q}=\left(\sum_{k \geq 1}\left|c_{k}\right|^{q}\right)^{1 / q}$.

Applying Kolmogorov's inequality and Theorem 1.2 to $P_{1}$, we have

$$
\begin{aligned}
\left(\frac{1}{|Q|} \int_{Q}\left|T_{q}\left(\vec{f}^{0}\right)(z)\right|^{\delta} d z\right)^{1 / \delta} & \leq C\left\|T_{q}\left(\vec{f}^{0}\right)\right\|_{L^{1 / m, \infty}\left(Q, \frac{d z}{|Q|}\right)} \\
& \leq C \prod_{j=1}^{m} \frac{1}{|Q|} \int_{Q}\left|f_{j}(z)\right|_{q_{j}} d z \\
& \leq C \prod_{j=1}^{m} M\left(\left|f_{j}\right|_{q_{j}}\right)(x) .
\end{aligned}
$$

We proceed to the estimate for $P_{2}$. We can take $t=[2 \sqrt{n} l(Q)]^{s}$. If $\alpha_{1}=\cdots=\alpha_{m}=\infty$, we have

$$
\begin{aligned}
\left(\frac{1}{|Q|} \int_{Q}|T(\vec{f})(z)-c|_{q}^{\delta} d z\right)^{1 / \delta} \\
\leq \frac{C}{|Q|} \int_{Q}\left|T\left(\overrightarrow{f^{\infty}}\right)(z)-c\right|_{q} d z \\
\leq \frac{C}{|Q|} \int_{Q}\left(\sum_{k=1}^{\infty}\left|T\left(\vec{f}_{k}^{\infty}\right)(z)-T\left(\vec{f}_{k}^{\infty}\right)(x)\right|^{q}\right)^{1 / q} d z \\
\leq \frac{C}{|Q|} \int_{Q}\left(\sum_{k=1}^{\infty}\left|\int_{\left(\mathbb{R}^{n} \backslash Q^{*}\right)^{m}}\right| K(z, \vec{y})-K(x, \vec{y})|| f_{1 k} \cdots f_{m k}|d \vec{y}|^{q}\right)^{1 / q} d z \\
\leq \frac{C}{|Q|} \int_{Q}\left(\sum_{k=1}^{\infty}\left|\int_{\left(\mathbb{R}^{n} \backslash Q^{*}\right)^{m}}\right| K(z, \vec{y})-K_{t}^{(0)}(z, \vec{y})|| f_{1 k} \cdots f_{m k}|d \vec{y}|^{q}\right)^{1 / q} d z \\
\quad+\frac{C}{|Q|} \int_{Q}\left(\sum_{k=1}^{\infty}\left|\int_{\left(\mathbb{R}^{n} \backslash Q^{*}\right)^{m}}\right| K_{t}^{(0)}(z, \vec{y})-K(x, \vec{y})|| f_{1 k} \cdots f_{m k}|d \vec{y}|^{q}\right)^{1 / q} d z \\
=P_{21}+P_{22} .
\end{aligned}
$$


Since $z \in Q$ and $y_{j} \in \mathbb{R}^{n} \backslash(8 \sqrt{n}+4) Q$, we get $\left|y_{j}-z\right|>(4 \sqrt{n}+1) l(Q)>2 t^{1 / s}$ for all $j=$ $1, \ldots, m$. Applying Assumption (H2), we obtain

$$
\begin{aligned}
P_{21} & \leq \frac{C}{|Q|} \int_{Q} \int_{\left(\mathbb{R}^{n} \backslash Q^{*}\right)^{m}} \frac{A t^{\varepsilon / s}}{\left(\left|z-y_{1}\right|+\cdots+\left|z-y_{m}\right|\right)^{m n+\varepsilon}}\left|f_{1}\right|_{q_{1}} \cdots\left|f_{m}\right|_{q_{m}} d \vec{y} d z \\
& \leq \sum_{k=1}^{\infty} \frac{1}{2^{k \varepsilon}} \prod_{j=1}^{m} \frac{1}{2^{(k+1) n}\left|Q^{*}\right|} \int_{2^{k+1} Q^{*}}\left|f_{j}\right|_{q_{j}} d y_{j} \\
& \leq C \prod_{j=1}^{m} M\left(\left|f_{j}\right|_{q_{j}}\right)(x) .
\end{aligned}
$$

Since $x, z \in Q,|z-x| \leq \sqrt{n} l(Q) \leq \frac{1}{2} t^{1 / s}$. Note that $\left|y_{j}-z\right|>(4 \sqrt{n}+1) l(Q)>2 t^{1 / s}$, for all $j=1, \ldots, m$, hence $\phi\left(\frac{\left|y_{j}-z\right|}{t^{1 / s}}\right)=0$. Similarly, we get $P_{22} \leq \prod_{j=1}^{m} M\left(\left|f_{j}\right|_{q_{j}}\right)(x)$.

Now we estimate the typical representative of $P_{2}$, that is, $\alpha_{1}=\cdots=\alpha_{l}=\infty$ and $\alpha_{l+1}=$ $\cdots=\alpha_{m}=0$.

$$
\begin{aligned}
\mid T & \left(f_{1}^{\infty}, \ldots, f_{l}^{\infty}, f_{l+1}^{0}, \ldots, f_{m}^{0}\right)(z)-\left.T\left(f_{1}^{\infty}, \ldots, f_{l}^{\infty}, f_{l+1}^{0}, \ldots, f_{m}^{0}\right)(x)\right|_{q} \\
\leq & \int_{\left(\mathbb{R}^{n}\right)^{m}}\left|K(z, \vec{y})-K_{t}^{(0)}(z, \vec{y})\right|+\left|K_{t}^{(0)}(z, \vec{y})-K(x, \vec{y})\right|\left|f_{1}\right|_{q_{1}} \cdots\left|f_{m}\right|_{q_{m}} d \vec{y} \\
= & \int_{\left(\mathbb{R}^{n}\right)^{m}}\left|K(z, \vec{y})-K_{t}^{(0)}(z, \vec{y})\right|\left|f_{1}\right|_{q_{1}} \cdots\left|f_{m}\right|_{q_{m}} d \vec{y} \\
& \quad+\int_{\left(\mathbb{R}^{n}\right)^{m}}\left|K_{t}^{(0)}(z, \vec{y})-K(x, \vec{y})\right|\left|f_{1}\right|_{q_{1}} \cdots\left|f_{m}\right|_{q_{m}} d \vec{y} .
\end{aligned}
$$

Thus, we get

$$
\begin{aligned}
\left(\frac{1}{|Q|} \int_{Q}\left|T\left(f_{1}^{\infty}, \ldots, f_{l}^{\infty}, f_{l+1}^{0}, \ldots, f_{m}^{0}\right)(z)-c\right|_{q}^{\delta} d z\right)^{1 / \delta} \\
\leq \frac{C}{|Q|} \int_{Q}\left|T\left(f_{1}^{\infty}, \ldots, f_{l}^{\infty}, f_{l+1}^{0}, \ldots, f_{m}^{0}\right)(z)-T\left(f_{1}^{\infty}, \ldots, f_{l}^{\infty}, f_{l+1}^{0}, \ldots, f_{m}^{0}\right)(x)\right|_{q} d z \\
\leq \frac{C}{|Q|} \int_{Q} \int_{\left(\mathbb{R}^{n}\right)^{m}}\left|K(z, \vec{y})-K_{t}^{(0)}(z, \vec{y})\right|\left|f_{1}\right|_{q_{1}} \cdots\left|f_{m}\right|_{q_{m}} d \vec{y} d z \\
\quad+\frac{C}{|Q|} \int_{Q} \int_{\left(\mathbb{R}^{n}\right)^{m}}\left|K_{t}^{(0)}(z, \vec{y})-K(x, \vec{y})\right|\left|f_{1}\right|_{q_{1}} \cdots\left|f_{m}\right|_{q_{m}} d \vec{y} d z \\
=P_{23}+P_{24} .
\end{aligned}
$$

For $P_{23}$, by Assumption (H2), we have

$$
\begin{aligned}
P_{23} \leq & \frac{C}{|Q|} \int_{Q}\left(\int_{\left(\mathbb{R}^{n} \backslash Q^{*}\right)^{l}} \frac{t^{\varepsilon / s} \prod_{j=1}^{l}\left|f_{j}\left(y_{j}\right)\right|_{q_{j}} d y_{j}}{\left(\sum_{j \in\{1,2, \ldots, l\}}\left|z-y_{j}\right|\right)^{m n+\varepsilon}}\right. \\
& \left.+\int_{\left(\mathbb{R}^{n} \backslash Q^{*}\right)^{l}} \frac{\prod_{j=1}^{l}\left|f_{j}\left(y_{j}\right)\right|_{q_{j}} d y_{j}}{\left(\sum_{j \in\{1,2, \ldots, l\}}\left|z-y_{j}\right|\right)^{m n}}\right) \prod_{j=l+1}^{m} \int_{Q^{*}}\left|f_{j}\left(y_{j}\right)\right|_{q_{j}} d y_{j} d z \\
\leq & \left(\sum_{k=1}^{\infty} \frac{\left|Q^{*}\right|^{\varepsilon / n}}{\left(2^{k}\left|Q^{*}\right|^{1 / n}\right)^{m n+\varepsilon}} \int_{\left(\mathbb{R}^{n} \backslash Q^{*}\right)^{l}} \prod_{j=1}^{l}\left|f_{j}\left(y_{j}\right)\right|_{q_{j}} d y_{j}\right.
\end{aligned}
$$




$$
\begin{aligned}
& \left.+\sum_{k=1}^{\infty} \frac{1}{\left(2^{k}\left|Q^{*}\right|^{1 / n}\right)^{m n}} \int_{\left(2^{k+1} Q^{*} \mid 2^{k} Q^{*}\right)^{l}} \prod_{j=1}^{l}\left|f_{j}\left(y_{j}\right)\right|_{q_{j}} d y_{j}\right) \prod_{j=l+1}^{m} \int_{Q^{*}}\left|f_{j}\left(y_{j}\right)\right|_{q_{j}} d y_{j} \\
\leq & C \prod_{j=1}^{m} M\left(\left|f_{j}\right| q_{j}\right)(x) .
\end{aligned}
$$

By a similar argument, we deduce that $P_{24} \leq C \prod_{j=1}^{m} M\left(\left|f_{j}\right|_{q_{j}}\right)(x)$. In other case, we can also deduce the same estimate with minor modifications on the above arguments. We have thus proved Lemma 3.2.

Lemma 3.3 Let $0<\delta<\varepsilon<1 / m, 1 / m<q<\infty$ and $1 / q=1 / q_{1}+\cdots+1 / q_{m}$ with $1<$ $q_{1}, \ldots, q_{m}<\infty$. Suppose that $\vec{b} \in(B M O)^{l}$. There then exists a constant $C>0$ depending only on $\delta$ and $\varepsilon$ such that

$$
\begin{aligned}
M_{\delta}^{\sharp}\left(T_{\Pi \vec{b}, q} \vec{f}\right)(x) \leq & C \prod_{j=1}^{l}\left\|b_{j}\right\|_{B M O}\left(\prod_{j=1}^{m} M_{L(\log L)}\left(\left|f_{j}\right|_{q_{j}}\right)(x)+M_{\varepsilon}\left(T_{q} \vec{f}\right)(x)\right) \\
& +C \sum_{j=1}^{l-1} \sum_{\sigma \in \mathcal{C}_{j}^{l}} \prod_{i \in \sigma}\left\|b_{i}\right\|_{B M O} M_{\varepsilon}\left(T_{\Pi b_{\sigma^{\prime}}, q} \vec{f}\right)(x)
\end{aligned}
$$

for any smooth vector function $\left\{\vec{f}_{k}\right\}_{k=1}^{\infty}$ for any $x \in \mathbb{R}^{n}$, where $\sigma^{\prime}=\{1, \ldots, l\} \backslash \sigma$.

Proof For simplicity of notation, we write $F(\vec{y})$ instead of the product of $m$ functions $f_{1}\left(y_{1}\right) \cdots f_{m}\left(y_{m}\right)$ and let $\lambda_{j}=\frac{1}{|2 Q|} \int_{2 Q} b_{j}(z) d z$, for $j=1, \ldots, l$. Let $x \in \mathbb{R}^{n}$ and $Q$ be a cube centered at $x$. Then we have

$$
\begin{aligned}
T_{\Pi \vec{b}}(\vec{f})(x)= & \int_{\left(\mathbb{R}^{n)^{m}}\right.}\left(b_{1}(x)-b_{1}\left(y_{1}\right)\right) \cdots\left(b_{l}(x)-b_{l}\left(y_{l}\right)\right) K(x, \vec{y}) F(\vec{y}) d \vec{y} \\
= & \int_{\left(\mathbb{R}^{n}\right)^{m}}\left(\left(b_{1}(x)-\lambda_{1}\right)-\left(b_{1}(y)-\lambda_{1}\right)\right) \cdots\left(\left(b_{l}(x)-\lambda_{l}\right)-\left(b_{l}(y)-\lambda_{l}\right)\right) \\
& \times K(x, \vec{y}) F(\vec{y}) d \vec{y} \\
= & \sum_{i=0}^{l} \sum_{\sigma \in \mathcal{C}_{i}^{l}}(-1)^{l-j} \prod_{j \in \sigma}\left(b_{j}(x)-\lambda_{j}\right) \int_{\left(\mathbb{R}^{n}\right)^{m}} \prod_{j \in \sigma^{\prime}}\left(b_{j}\left(y_{j}\right)-\lambda_{j}\right) K(x, \vec{y}) F(\vec{y}) d \vec{y} \\
= & \left(b_{1}(x)-\lambda_{1}\right) \cdots\left(b_{l}(x)-\lambda_{l}\right) T(\vec{f})(x)+T\left(\left(b_{1}\left(\cdot_{1}\right)-\lambda_{1}\right) \cdots\left(b_{l}(\cdot \cdot)-\lambda_{l}\right) \vec{f}\right)(x) \\
& +\sum_{i=1}^{l-1} \sum_{\sigma \in \mathcal{C}_{i}^{l}}(-1)^{l-j} \prod_{j \in \sigma}\left(b_{j}(x)-\lambda_{j}\right) \int_{\left(\mathbb{R}^{n}\right)^{m}} \prod_{j \in \sigma^{\prime}}\left(b_{j}\left(y_{j}\right)-\lambda_{j}\right) K(x, \vec{y}) F(\vec{y}) d \vec{y} .
\end{aligned}
$$

Noting that $\prod_{j \in \sigma^{\prime}}\left(b_{j}\left(y_{j}\right)-\lambda_{j}\right)=\prod_{j \in \sigma^{\prime}}\left[\left(b_{j}\left(y_{j}\right)-b_{j}(x)\right)-\left(b_{j}(x)-\lambda_{j}\right)\right]$. Then we get

$$
\begin{aligned}
T_{\Pi \vec{b}, q} \vec{f}(x) \leq & \left|\left(b_{1}(x)-\lambda_{1}\right) \cdots\left(b_{l}(x)-\lambda_{l}\right)\right| T_{q}(\vec{f})(x) \\
& +\left|T\left(\left(b_{1}\left(\cdot_{1}\right)-\lambda_{1}\right) \cdots\left(b_{l}\left(\cdot{ }_{l}\right)-\lambda_{l}\right) \vec{f}\right)(x)\right|_{q} \\
& +C \sum_{i=1}^{l-1} \sum_{\sigma \in \mathcal{C}_{i}^{l}} \prod_{j \in \sigma}\left|b_{j}(x)-\lambda_{j}\right| T_{\Pi b_{\sigma^{\prime}, q}} \vec{f}(x) .
\end{aligned}
$$


Since $0<\delta<1 / m<1$, it follows that

$$
\begin{aligned}
\left(\left.\frac{1}{|Q|} \int_{Q}|| T_{\Pi \vec{b}, q}(\vec{f})(z)\right|^{\delta}-|\mathcal{C}|^{\delta} \mid d z\right)^{1 / \delta} & \\
\leq & C\left(\frac{1}{|Q|} \int_{Q}\left|T_{\Pi \vec{b}}(\vec{f})(z)-c\right|_{q}^{\delta} d z\right)^{1 / \delta} \\
\leq & C\left(\left.\frac{1}{|Q|} \int_{Q}\left|\left(b_{1}(z)-\lambda_{1}\right) \cdots\left(b_{l}(z)-\lambda_{l}\right)\right| T(\vec{f})(z)\right|_{q} ^{\delta} \mid d z\right)^{1 / \delta} \\
& +C \sum_{i=1}^{l-1} \sum_{\sigma \in \mathcal{C}_{i}^{l}}\left(\frac{1}{|Q|} \int_{Q} \prod_{j \in \sigma}\left(\left|b_{j}(z)-\lambda_{j}\right| T_{\Pi b_{\sigma^{\prime}, q}} \vec{f}(z)\right)^{\delta} d z\right)^{1 / \delta} \\
& +C\left(\frac{1}{|Q|} \int_{Q}\left|T\left(\left(b_{1}\left(\cdot \cdot_{1}\right)-\lambda_{1}\right) \cdots\left(b_{l}(\cdot l)-\lambda_{l}\right) \vec{f}\right)(z)-c\right|_{q}^{\delta} d z\right)^{1 / \delta} \\
\triangleq & I I+I I I,
\end{aligned}
$$

where $\mathcal{C}=|c|_{q}=\left(\sum_{k \geq 1}\left|c_{k}\right|^{q}\right)^{1 / q}$. We can choose $1<p_{1}, \ldots, p_{l}<\infty$ with $\frac{1}{p_{1}}+\cdots+\frac{1}{p_{l}}+\frac{1}{\varepsilon}=\frac{1}{\delta}$. Since $0<\delta<\varepsilon<1 / m$, Hölder's inequality gives

$$
\begin{aligned}
& I \leq C \prod_{j=1}^{l}\left\|b_{j}\right\|_{B M O} M_{\varepsilon}\left(T_{q} \vec{f}\right)(x), \\
& I I \leq C \sum_{i=1}^{l-1} \sum_{\sigma \in \mathcal{C}_{i}^{l}} \prod_{j \in \sigma}\left\|b_{j}\right\|_{B M O} M_{\varepsilon}\left(T_{\Pi b_{\sigma^{\prime}}, q} \vec{f}\right)(x) .
\end{aligned}
$$

Let us estimate term III. Set $\vec{f}_{j}=\vec{f}_{j}^{0}+\vec{f}_{j}^{\infty}$, where $\vec{f}_{j}^{0}=\vec{f}_{j} \chi_{Q^{*}}$. Let $\vec{f}^{\alpha}=f_{1}^{\alpha_{1}} \cdots f_{m}^{\alpha_{m}}$ and $Q^{*}=$ $(8 \sqrt{n}+4) Q$. Taking $\mathcal{C}=\sum_{\alpha_{1}, \ldots, \alpha_{m}}\left|T\left(\left(b_{1}\left(\cdot \cdot_{1}\right)-\lambda_{1}\right) \cdots\left(b_{l}\left(\cdot \cdot_{l}\right)-\lambda_{l}\right) f_{1}^{\alpha_{1}} \cdots f_{m}^{\alpha_{m}}\right)(x)\right|_{q}$, we have

$$
\begin{aligned}
& \left|T_{\Pi \vec{b}, q}(\vec{f})(z)-\mathcal{C}\right| \\
& \quad \leq T_{q}\left(\left(b_{1}\left(\cdot \cdot_{1}\right)-\lambda_{1}\right) \cdots\left(b_{l}(\cdot l)-\lambda_{l}\right) \vec{f}^{0}\right)(z) \\
& \quad+C \sum_{\alpha_{1}, \ldots, \alpha_{m}} \mid\left(T\left(\left(b_{1}\left(\cdot \cdot_{1}\right)-\lambda_{1}\right) \cdots\left(b_{l}(\cdot l)-\lambda_{l}\right) \vec{f}^{\alpha}\right)\right)(z) \\
& \quad-\left.\left(T\left(\left(b_{1}\left(\cdot \cdot_{1}\right)-\lambda_{1}\right) \cdots\left(b_{l}\left(\cdot \cdot_{l}\right)-\lambda_{l}\right) \vec{f}^{\alpha}\right)\right)(x)\right|_{q}
\end{aligned}
$$

where in the last sum each $\alpha_{j}=0$ or $\infty$ and in each term there is at least one $\alpha_{j}=\infty$.

If $\alpha_{1}=\cdots=\alpha_{m}=0$, applying Kolmogorov's inequality and Theorem 1.2 , we get

$$
\begin{aligned}
& \left(\frac{1}{|Q|} \int_{Q}\left|T_{q}\left(\left(b_{1}\left(\cdot_{1}\right)-\lambda_{1}\right) \cdots\left(b_{l}(\cdot \cdot)-\lambda_{l}\right) \vec{f}^{0}\right)(z)\right|^{\delta} d z\right)^{1 / \delta} \\
& \quad \leq C\left\|T_{q}\left(\left(b_{1}\left(\cdot_{1}\right)-\lambda_{1}\right) \cdots\left(b_{l}(\cdot l)-\lambda_{l}\right) \vec{f}^{0}\right)\right\|_{L^{1 / m, \infty}\left(Q, \frac{d z}{|Q|}\right)} \\
& \quad \leq C \prod_{j=1}^{l} \frac{1}{|Q|} \int_{Q}\left|b_{j}\left(y_{j}\right)-\lambda_{j}\right|\left|f_{j}(z)\right|_{q_{j}} d z \prod_{j=l+1}^{m} \frac{1}{|Q|} \int_{Q}\left|f_{j}(z)\right|_{q_{j}} d z
\end{aligned}
$$




$$
\begin{aligned}
& \leq C \prod_{j=1}^{l}\left\|b_{j}\right\|_{B M O}\left\|\left|f_{j}\right|_{q_{j}}\right\|_{L(\log L), Q} \prod_{j=l+1}^{m} \frac{1}{|Q|} \int_{Q}\left|f_{j}(z)\right|_{q_{j}} d z \\
& \leq C \prod_{j=1}^{l}\left\|b_{j}\right\|_{B M O} \prod_{j=1}^{m} M_{L(\log L)}\left(\left|f_{j}\right|_{q_{j}}\right)(x) .
\end{aligned}
$$

If $\alpha_{1}=\cdots=\alpha_{m}=\infty$, we have

$$
\begin{aligned}
\left(\frac{1}{|Q|} \int_{Q}\left|T_{\Pi \vec{b}}\left(\vec{f}^{\infty}\right)(z)-c\right|_{q}^{\delta} d z\right)^{1 / \delta} \\
\leq \frac{C}{|Q|} \int_{Q}\left|T_{\Pi \vec{b}}\left(\vec{f}^{\infty}\right)(z)-c\right|_{q} d z \\
\leq \frac{C}{|Q|} \int_{Q}\left(\sum_{k=1}^{\infty}\left|T_{\Pi \vec{b}}\left(\vec{f}_{k}^{\infty}\right)(z)-T_{\Pi \vec{b}}\left(\vec{f}_{k}^{\infty}\right)(x)\right|^{q}\right)^{1 / q} d z \\
\leq \frac{C}{|Q|} \int_{Q}\left(\sum_{k=1}^{\infty}\left|\int_{\left(\mathbb{R}^{n} \backslash Q^{*}\right)^{m}}\right| K(z, \vec{y})-K(x, \vec{y}) \mid\right. \\
\left.\quad \times\left.\left|\left(b_{1}\left(y_{1}\right)-\lambda_{1}\right) \cdots\left(b_{l}\left(y_{l}\right)-\lambda_{l}\right) f_{1 k} \cdots f_{m k}\right| d \vec{y}\right|^{q}\right)^{1 / q} d z \\
\leq \frac{C}{|Q|} \int_{Q}\left(\sum_{k=1}^{\infty}\left|\int_{\left(\mathbb{R}^{n} \backslash Q^{*}\right)^{m}}\right| K(z, \vec{y})-K_{t}^{(0)}(z, \vec{y}) \mid\right. \\
\left.\quad \times\left.\left|\left(b_{1}\left(y_{1}\right)-\lambda_{1}\right) \cdots\left(b_{l}\left(y_{l}\right)-\lambda_{l}\right) f_{1 k} \cdots f_{m k}\right| d \vec{y}\right|^{q}\right)^{1 / q} d z \\
\quad+\frac{C}{|Q|} \int_{Q}\left(\sum_{k=1}^{\infty}\left|\int_{\left(\mathbb{R}^{n} \backslash Q^{*}\right)^{m}}\right| K_{t}^{(0)}(z, \vec{y})-K(x, \vec{y}) \mid\right. \\
\left.\quad \times\left.\left|\left(b_{1}\left(y_{1}\right)-\lambda_{1}\right) \cdots\left(b_{l}\left(y_{l}\right)-\lambda_{l}\right) f_{1 k} \cdots f_{m k}\right| d \vec{y}\right|^{q}\right)^{1 / q} d z \\
=I I I_{1}+I I I_{2} .
\end{aligned}
$$

Consider now the term $I I I_{1}$. Taking $t=[2 \sqrt{n} l(Q)]^{s}$, we have by Assumption (H2)

$$
\begin{aligned}
I I I_{1} \leq \leq & \frac{C}{|Q|} \int_{Q} \int_{\left(\mathbb{R}^{n} \backslash Q^{*}\right)^{m}} \sum_{k=1}^{\infty} \frac{\left|Q^{*}\right|^{\varepsilon / n}}{\left(2^{k}\left|Q^{*}\right|^{1 / n}\right)^{m n+\varepsilon}} \\
& \times\left|\left(b_{1}\left(y_{1}\right)-\lambda_{1}\right) \cdots\left(b_{l}\left(y_{l}\right)-\lambda_{l}\right)\right|\left|f_{1}\right|_{q_{1}} \cdots\left|f_{m}\right|_{q_{m}} d \vec{y} d z \\
\leq & C \prod_{j=1}^{l} \sum_{k=1}^{\infty} \frac{1}{2^{k \varepsilon}} \frac{1}{2^{(k+1) n}\left|Q^{*}\right|} \\
& \times \int_{2^{k+1} Q^{*}}\left|b_{j}\left(y_{j}\right)-\lambda_{j}\right|\left|f_{j}\right| q_{j} d y_{j} \prod_{j=l+1}^{m} \frac{1}{2^{(k+1) n}\left|Q^{*}\right|} \int_{2^{k+1} Q^{*}}\left|f_{j}\right|_{q_{j}} d y_{j} \\
\leq & C \prod_{j=1}^{l} \sum_{k=1}^{\infty} \frac{k}{2^{k \varepsilon}}\left\|b_{j}\right\|_{B M O}\left\|\left|f_{j}\right|_{q_{j}}\right\|_{L(\log L), 2^{k+1} Q^{*}} \prod_{j=l+1}^{m} \frac{1}{2^{(k+1) n}\left|Q^{*}\right|} \int_{2^{k+1} Q^{*}}\left|f_{j}\right|_{q_{j}} d y_{j}
\end{aligned}
$$




$$
\begin{aligned}
& \leq C \prod_{j=1}^{l}\left\|b_{j}\right\|_{B M O}\left\|\left|f_{j}\right|_{q_{j}}\right\|_{L(\log L), 2^{k+1} Q^{*}} \prod_{j=l+1}^{m} \frac{1}{2^{(k+1) n}\left|Q^{*}\right|} \int_{2^{k+1} Q^{*}}\left|f_{j}\right|_{q_{j}} d y_{j} \\
& \leq C \prod_{j=1}^{l}\left\|b_{j}\right\|_{B M O} \prod_{j=1}^{m} M_{L(\log L)}\left(\left|f_{j}\right|_{q_{j}}\right)(x) .
\end{aligned}
$$

Similarly, we get $I I I_{2} \leq \prod_{j=1}^{l}\left\|b_{j}\right\|_{B M O} \prod_{j=1}^{m} M_{L(\log L)}\left(\left|f_{j}\right|_{q_{j}}\right)(x)$. Now we consider only the typical representative of III. Similar to the estimates of $P_{2}$ in Lemma 3.2, we have

$$
\begin{aligned}
&\left(\frac{1}{|Q|} \int_{Q}\left|T\left(\left(b_{1}(\cdot \cdot 1)-\lambda_{1}\right) \cdots\left(b_{l}(\cdot \cdot)-\lambda_{l}\right) f_{1}^{\infty}, \ldots, f_{l}^{\infty}, f_{l+1}^{0}, \ldots, f_{m}^{0}\right)(z)-c\right|_{q}^{\delta} d z\right)^{1 / \delta} \\
& \leq \frac{C}{|Q|} \int_{Q} \mid T\left(\left(b_{1}(\cdot \cdot 1)-\lambda_{1}\right) \cdots\left(b_{l}(\cdot \cdot)-\lambda_{l}\right) f_{1}^{\infty}, \ldots, f_{l}^{\infty}, f_{l+1}^{0}, \ldots, f_{m}^{0}\right)(z) \\
& \quad-\left.T\left(\left(b_{1}(\cdot \cdot 1)-\lambda_{1}\right) \cdots\left(b_{l}(\cdot l)-\lambda_{l}\right) f_{1}^{\infty}, \ldots, f_{l}^{\infty}, f_{l+1}^{0}, \ldots, f_{m}^{0}\right)(x)\right|_{q} d z \\
& \leq \prod_{j=1}^{l}\left(\int_{\left(\mathbb{R}^{n} \backslash Q^{*}\right)^{l}} \frac{t^{\varepsilon / s}\left|\left(b_{1}\left(y_{1}\right)-\lambda_{1}\right) \cdots\left(b_{l}\left(y_{l}\right)-\lambda_{l}\right)\right| \prod_{j=1}^{l}\left|f_{j}\left(y_{j}\right)\right|_{q_{j}} d y_{j}}{\left(\sum_{j \in\{1,2, \ldots, l\}}\left|z-y_{j}\right|\right)^{m n+\varepsilon}}\right. \\
& \quad+\int_{\left(\mathbb{R}^{n} \backslash Q^{*}\right)^{l}} \frac{\left|\left(b_{1}\left(y_{1}\right)-\lambda_{1}\right) \cdots\left(b_{l}\left(y_{l}\right)-\lambda_{l}\right)\right| \prod_{j=1}^{l}\left|f_{j}\left(y_{j}\right)\right|_{q_{j}} d y_{j}}{\left(\sum_{j \in\{1,2, \ldots, l\}}\left|z-y_{j}\right|\right)^{m n}} \prod_{j=l+1}^{m} \int_{Q^{*}}\left|f_{j}\left(y_{j}\right)\right|_{q_{j}} d y_{j} \\
& \leq C \prod_{j=1}^{l}\left\|b_{j}\right\|_{B M O}\left\|\left|f_{j}\right|_{q_{j}}\right\|_{L(\log L), 2^{k+1} Q^{*}} \prod_{j=l+1}^{m} \frac{1}{\left|Q^{*}\right|} \int_{Q^{*}}\left|f_{j}(z)\right|_{q_{j}} d z \\
& \leq C \prod_{j=1}^{l}\left\|b_{j}\right\|_{B M O} \prod_{j=1}^{m} M_{L(\log L)}\left(\left|f_{j}\right|_{q_{j}}\right)(x) .
\end{aligned}
$$

Then Lemma 3.3 is proved.

Lemma 3.4 Let $0<p<\infty, 1 / m<q<\infty$, and $\frac{1}{q_{1}}+\cdots+\frac{1}{q_{m}}=\frac{1}{q}$ with $1<q_{1}, \ldots, q_{m}<\infty$ and let $w \in A_{\infty}$. Suppose that $\vec{b} \in(B M O)^{l}$. Then there exists a constant $C>0$ such that

$$
\int_{\mathbb{R}^{n}}\left|T_{\Pi \vec{b}, q} \vec{f}\right|^{p} w(x) d x \leq C \prod_{j=1}^{l}\left\|b_{j}\right\|_{B M O}^{p} \int_{\mathbb{R}^{n}}\left(\prod_{j=1}^{m} M_{L(\log L)}\left(\left|f_{j}\right|_{q_{j}}\right)(x)\right)^{p} w(x) d x
$$

for any smooth function $\vec{f}$ with compact support.

Proof We assume that the right-hand side of (3.2) is finite, since otherwise there is nothing to be proved. For $l=1$, by using Lemma 3.1 and Lemma 3.3, we obtain

$$
\begin{aligned}
\left\|T_{\Pi \vec{b}, q} \vec{f}\right\|_{L^{p}(\omega)} & \leq\left\|M_{\delta} T_{\Pi \vec{b}, q} \vec{f}\right\|_{L^{p}(\omega)} \leq C\left\|M_{\delta}^{\sharp} T_{\Pi \vec{b}, q} \vec{f}\right\|_{L^{p}(\omega)} \\
& \leq C\left\|b_{1}\right\|_{B M O}\left[\left\|M_{\varepsilon}\left(T_{q} \vec{f}\right)\right\|_{L^{p}(\omega)}+\left\|\prod_{j=1}^{m} M_{L(\log L)}\left(\left|f_{j}\right|_{q_{j}}\right)\right\|_{L^{p}(\omega)}\right] \\
& \leq C\left\|b_{1}\right\|_{B M O}\left[\left\|T_{q} \vec{f}\right\|_{L^{p}(\omega)}+\left\|\prod_{j=1}^{m} M_{L(\log L)}\left(\left|f_{j}\right|_{q_{j}}\right)\right\|_{L^{p}(\omega)}\right]
\end{aligned}
$$




$$
\begin{aligned}
& \leq C\left\|b_{1}\right\|_{B M O}\left[\left\|\prod_{j=1}^{m} M\left(\left|f_{j}\right|_{q_{j}}\right)\right\|_{L^{p}(\omega)}+\left\|\prod_{j=1}^{m} M_{L(\log L)}\left(\left.\left|f_{j}\right|\right|_{q_{j}}\right)\right\|_{L^{p}(\omega)}\right] \\
& \leq C\left\|b_{1}\right\|_{B M O}\left\|\prod_{j=1}^{m} M_{L(\log L)}\left(\left|f_{j}\right|_{q_{j}}\right)\right\|_{L^{p}(\omega)} .
\end{aligned}
$$

For the general case $l \geq 2$, similarly to the case for $l=1$, we have

$$
\left\|T_{\Pi \vec{b}, \vec{q}} \vec{f}\right\|_{L^{p}(\omega)} \leq C \prod_{j=1}^{l}\left\|b_{j}\right\|_{B M O}\left\|\prod_{j=1}^{m} M_{L(\log L)}\left(\left|f_{j}\right|_{q_{j}}\right)\right\|_{L^{p}(\omega)} .
$$

To apply the Fefferman-Stein inequality in (3.3), one needs to verify now that $\left\|T_{\Pi \vec{b}, q} \vec{f}\right\|_{L^{p}(\omega)}<\infty$ and $\left\|T_{q} \vec{f}\right\|_{L^{p}(\omega)}<\infty$. We will only show the first one since the proof of another one is very similar but easier.

Suppose that the symbols $b_{j}$ and the weight $\omega$ are bounded functions. Since $\vec{f}$ has compact support, we may assume $\operatorname{supp} f_{j} \subset B(0, R)$. Then we have

$$
\begin{aligned}
\left\|T_{\Pi \vec{b}, q} \vec{f}\right\|_{L^{p}(\omega)} & =\int_{|x| \leq 2 R}\left|T_{\Pi \vec{b}, q} \vec{f}(x)\right|^{p} \omega(x) d x+\int_{|x|>2 R}\left|T_{\Pi \vec{b}, q} \vec{f}(x)\right|^{p} \omega(x) d x \\
& =I_{1}+I_{2} .
\end{aligned}
$$

We choose $s>p$ and $s_{1}, \ldots, s_{m}>1$ such that $1 / s=1 / s_{1}+\cdots+1 / s_{m}$. Theorem 1.1 and Hölder's inequality imply

$$
\begin{aligned}
I_{1} & \leq C \int_{|x| \leq 2 R}\left|T_{\Pi \vec{b}, q} \vec{f}\right|^{p} d x \leq C\left(\int_{\mathbb{R}^{n}}\left|T_{q} \vec{f}\right|^{s} d x\right)^{p / s} R^{n(s-p) / s} \\
& \leq C\left(\prod_{j=1}^{m}\left\|\left|f_{j}\right|_{q_{j}}\right\|_{L^{s_{j}}}\right)^{p} R^{n(s-p) / s}<\infty .
\end{aligned}
$$

Consider now the term $I_{2}$. Since $|x|>2 R$, we have by Assumption (H2)

$$
\begin{aligned}
\left|T_{\Pi \vec{b}, q} \vec{f}(x)\right| \leq & C\left|\int_{\left(B(0,2 R)^{m}\right.}\right| K(x, \vec{y})|| b_{1}(x)-b_{1}\left(y_{1}\right)|\cdots| b_{l}(x)-b_{l}\left(y_{l}\right) \mid \\
& \times\left.\left|f_{1}\left(y_{1}\right)\right| \cdots\left|f_{m}\left(y_{m}\right)\right| d \vec{y}\right|_{q} \\
\leq & C \int_{(B(0,2 R))^{m}}\left|K(x, \vec{y})-K_{t}^{(0)}(x, \vec{y})\right| \prod_{j=1}^{m}\left|f_{j}\left(y_{j}\right)\right|_{q_{j}} d \vec{y} \\
& +\int_{(B(0,2 R))^{m}}\left|K_{t}^{(0)}(x, \vec{y})\right| \prod_{j=1}^{m}\left|f_{j}\left(y_{j}\right)\right|_{q_{j}} d \vec{y} \\
\leq & C \prod_{j=1}^{m} \frac{1}{|x|^{n}} \int_{B(0,|x|)}\left|f_{j}\left(y_{j}\right)\right|_{q_{j}} d y_{j} \leq \prod_{j=1}^{m} M_{L(\log L)}\left(\left|f_{j}\right|_{q_{j}}\right)(x) .
\end{aligned}
$$

Thus, $I_{2} \leq C \int_{\mathbb{R}^{n}}\left(\prod_{j=1}^{m} M_{L(\log L)}\left(\left|f_{j}\right|_{q_{j}}\right)(x)\right)^{p} w(x) d x<\infty$.

For the general case, we can check the limit (see the proof of [10], Theorem 1.1, we omit the details here). Thus, (3.2) is proved. 
Proof of Theorem 1.3 (i) Since $v_{\vec{\omega}} \in A_{m p} \subset A_{\infty}$, Lemma 3.4 gives

$$
\int_{R^{n}}\left|T_{\Pi \vec{b}, q}(\vec{f})(x)\right|^{p} v_{\vec{\omega}} d x \leq C \int_{R^{n}} \prod_{j=1}^{m} M_{L(\log L)}^{p}\left(\left|f_{j}\right|_{q_{j}}\right)(x) v_{\vec{\omega}} d x
$$

It follows from [8] that there exists $r>1$ such that $v_{\vec{\omega}} \in A_{\left(\frac{p_{1}}{r}, \ldots, \frac{p_{m}}{r}\right)}$. On the other hand, since $\Phi(t)=t\left(1+\log ^{+} t\right) \leq t^{r}$ for all $t>1$, the generalized Jensen inequality yields that

$$
\left\|f_{j}\right\|_{L(\log L), Q} \leq C\left(\frac{1}{|Q|} \int_{Q}\left|f_{j}(y)\right|^{r} d y\right)^{1 / r}
$$

for all $j$. One sees immediately that

$$
\left\|T_{\Pi \vec{b}, q}(\vec{f})\right\|_{L^{p}\left(v_{\vec{\omega}}\right)} \leq C\left\|\prod_{j=1}^{m} M_{r}\left(\left|f_{j}\right|_{q_{j}}\right)\right\|_{L^{p}\left(v_{\vec{\omega}}\right)}
$$

Since $v_{\vec{\omega}} \in A_{\left(\frac{p_{1}}{r}, \ldots, \frac{p_{m}}{r}\right)}$, it follows from Hölder's inequality and the well-known inequality of Fefferman-Stein [9] that

$$
\left\|\prod_{j=1}^{m} M\left(\left|f_{j}\right|_{q_{j}}\right)\right\|_{L^{p / r}\left(v_{\vec{\omega}}\right)} \leq C \prod_{j=1}^{m}\left\|\left|f_{j}\right|_{q_{j}}\right\|_{L^{p_{j} / r}\left(M \omega_{j}\right)}
$$

We then have

$$
\left\|T_{\Pi \vec{b}, q}(\vec{f})\right\|_{L^{p}\left(v_{\vec{\omega}}\right)} \leq C \prod_{j=1}^{l}\left\|b_{j}\right\|_{B M O} \prod_{j=1}^{m}\left\|\left|f_{j}\right|_{q_{j}}\right\|_{L^{p_{j}}\left(M \omega_{j}\right)} .
$$

This is the desired conclusion.

(ii) Since $\omega_{j} \in A_{p_{j}}$, there exists $r>0$ such that $\omega_{j} \in A_{p_{j} / r}$. Analysis similar to that in the proof of Theorem 1.3 (i) shows that

$$
\left\|T_{\Pi \vec{b}, q}(\vec{f})\right\|_{L^{p}\left(v_{\vec{\omega}}\right)} \leq C\left\|\prod_{j=1}^{m} M_{r}\left(\left|f_{j}\right|_{q_{j}}\right)\right\|_{L^{p}\left(v_{\vec{\omega}}\right)}
$$

The Hölder inequality implies

$$
\begin{aligned}
\left\|\prod_{j=1}^{m} M_{r}\left(\left|f_{j}\right|_{q_{j}}\right)\right\|_{L^{p}\left(v_{\vec{\omega}}\right)} & =\left(\left\|\prod_{j=1}^{m} M\left(\left|f_{j}\right|_{q_{j}}^{r}\right)\right\|_{L^{p / r}\left(v_{\vec{\omega}}\right)}\right)^{1 / r} \\
& \leq\left(\prod_{j=1}^{m}\left\|M\left(\left|f_{j}\right|_{q_{j}}^{r}\right)\right\|_{L^{p_{j} / r}(\omega)}\right)^{1 / r} \\
& \leq \prod_{j=1}^{m}\left\|\left|f_{j}\right|_{q_{j}}\right\|_{L^{p_{j}}\left(\omega_{j}\right)^{.}}
\end{aligned}
$$

To prove Theorem 1.3 holds for $T^{*}$, it suffices to prove Lemmas 3.2-3.4 hold for $T^{*}$. The proof follows from similar steps in [11] and combines the argument we used in the 
above lemmas. The key for tackling the new complexities is a very careful deal with the supremum, we refer the reader to [11]. This concludes the proof of the theorem.

\section{Competing interests}

The author declares that he has no competing interests.

\section{Acknowledgements}

This work was supported by the Mathematical Tianyuan Foundation of China (No. 11226102). The author thanks the referees for carefully reading the manuscript and providing many valuable suggestions, which have improved this article.

Received: 11 March 2013 Accepted: 30 April 2013 Published: 17 May 2013

\section{References}

1. Grafakos, L, Torres, R: Multilinear Calderón-Zygmund theory. Adv. Math. 165, 124-164 (2002)

2. Grafakos, L, Torres, RH: Maximal operator and weighted norm inequalities for multilinear singular integrals. Indiana Univ. Math. J. 51, 1261-1276 (2002)

3. Grafakos, L, Martell, JM: Extrapolation of weighted norm inequalities for multivariable operators. J. Geom. Anal. 14 $19-46(2004)$

4. Cruz-Uribe, D, Martell, JM, Pérez, C: Extrapolation from $A_{\infty}$ weights and applications. J. Funct. Anal. 213, 412-439 (2004)

5. Peng, X, Si, Z, Xue, Q: Weighted estimates for iterated commutators of multilinear operators with non-smooth kernels. Math. Inequal. Appl. (to appear)

6. Anh, BT, Duong, XT: On commutators of vector BMO functions and multilinear singular integrals with non-smooth kernels. J. Math. Anal. Appl. 371, 80-84 (2010)

7. Grafakos, L, Liu, L, Yang, D: Multiple weighted norm inequalities for maximal multilinear singular integrals with non-smooth kernels. Proc. R. Soc. Edinb., Sect. A 141, 755-775 (2011)

8. Lerner, A, Ombrosi, S, Pérez, C, Torres, RH, Trujillo-González, R: New maximal functions and multiple weights for the multilinear Calderón-Zygmund theory. Adv. Math. 220, 1222-1264 (2009)

9. Fefferman, C, Stein, E: $H^{p}$ spaces of several variables. Acta Math. 129, 137-193 (1972)

10. Pérez, C, Trujillo-Gonzalez, R: Sharp weighted estimates for multilinear commutators. J. Lond. Math. Soc. (2) 65 , 672-692 (2002)

11. $\mathrm{Si}, \mathrm{Z}, \mathrm{Xue}, \mathrm{Q}$ : Weighted estimates for commutators of vector-valued maximal multilinear operators. Preprint

doi:10.1186/1029-242X-2013-250

Cite this article as: Si: Weighted estimates for vector-valued multilinear operators with non-smooth kernels. Journal of Inequalities and Applications 2013 2013:250.

\section{Submit your manuscript to a SpringerOpen ${ }^{\circ}$ journal and benefit from:}

- Convenient online submission

- Rigorous peer review

- Immediate publication on acceptance

Open access: articles freely available online

- High visibility within the field

- Retaining the copyright to your article 